\title{
Influence de la fertilisation sur la capacité de nodulation de deux espèces de legumineuses, Vigna radiata L.Wilczek et Vigna unguiculata L.Walp (Fabaceae)
}

\author{
Jacob N'dri KOUASSI*, N'guessan KOUAME, Koutoua AYOLIE, Joël Koffi YAO et \\ Justin Kouadio YATTY
}
Université Jean Lorougnon GUEDE, Laboratoire d'Amélioration de la Production Agricole, UFR Agroforesterie, BP 150 Daloa, Côte d'Ivoire.
*Auteur correspondant; E-mail: kouassindrijacob@yahoo.fr; Tel: (+225) 48667448.

\section{RESUME}

La présente étude a été conduite afin d'accroitre la capacité de nodulation des légumineuses. Pour ce faire, un dispositif de 6 traitements avec 3 répétitions a été mis en place. Il s'agit des traitements T1: sans apport de fertilisants; T2: apport d'azote sous forme d'urée; T3: apport de phosphore sous forme de triple superphosphate; T4: apport d'un mélange azote-phosphate dans les mêmes proportions;T5 : apport d'engrais complexe NPK; T6: sol amendé avec de l'engrais organique sous forme de compost. L'évaluation de la capacité de nodulation a été faite en considérant deux paramètres après la floraison. Il s'agit du nombre et du poids des nodules. Les résultats de l'analyse statistique ont montré que contrairement au type de fertilisant, l'espèce de légumineuse n'influence pas la nodulation. Ainsi, le nombre et le poids de nodule les plus élevés sont obtenus avec la fertilisation organique. En outre, cette présente étude pourrait être étendue sur d'autres légumineuses

(C) 2019 International Formulae Group. All rights reserved

Mots clés : fertilisant, compost, azote phosphore, bactéries rhizobia

\section{Influence of fertilization on the nodulation capacity of two legume species, Vigna radiata L.Wilczek and Vigna unguiculata L.Walp (Fabaceae)}

\begin{abstract}
This study was conducted to increase the nodulation capacity of legumes. To do this, a device of 6 treatments with 3 repetitions has been set up. These are T1 treatments: without fertilizer; T2: nitrogen supply in the form of urea; T3: phosphorus supply as triple superphosphate; T4: supply of a nitrogen-phosphate mixture in the same proportions, T5: complex NPK fertilizer input; T6: Soil amended with organic fertilizer in the form of compost. The evaluation of the nodulation capacity was made by considering two parameters after flowering. This is the number and weight of the nodules. The results of the statistical analysis showed that unlike the type of fertilizer, the leguminous species does not influence nodulation. Thus, the highest number and weight of nodules are obtained with organic fertilization. In addition, this present study could be extended to other legumes.

(C) 2019 International Formulae Group. All rights reserved
\end{abstract}

Keywords: Fertilizer, compost, phosphorus rhizobia, bacteria. 


\section{INTRODUCTION}

La dégradation des sols et particulièrement la baisse de la fertilité chimique due à l'appauvrissement des sols en matière organique, en azote et autres éléments minéraux, est connue de tous les spécialistes comme étant à la base de la chute de productivité agricole en Afrique tropicale (Kasongo et al., 2012). Par ailleurs, les systèmes de productions traditionnelles acidifient souvent les sols agricoles dans les pays en voie de développement. Pour y faire face, les systèmes agricoles traditionnels utilisent souvent la rotation " jachère-culture de subsistance », pour restaurer la fertilité du sol (Floret et Pontanier, 2000). Aussi, la fertilisation chimique (engrais azotés) envisagée comme alternative, a permis durant plusieurs décennies, d'augmenter les productions agricoles. Cependant, elle est très souvent inappropriée (coût élevé, destruction de la microfaune et microfaune du sol et pollution des eaux) (Graveline et Loubier, 2004). Face à cette situation, il est urgent de développer des techniques de fertilisation rationnelle, efficiente et accessible aux producteurs. Ces systèmes doivent être économiquement satisfaisants pour les agriculteurs, avec de faibles impacts sur l'environnement. Un des rôles de la recherche agronomique est de fournir des bases scientifiques pour concevoir ces systèmes de culture, afin d'améliorer la productivité agricole mondiale et en particulier celles des pays d'Afrique. Ceci dans un concept d'une agriculture respectueuse des concepts de durabilité. Parmi les solutions envisageables, les légumineuses paraissent comme une alternative d'intérêt important du fait de leur capacité d'enrichissement rapide des sols par la fixation d'azote atmosphérique et la production abondante de biomasse végétale. Cette fixation est due à la présence de bactéries appartenant aux rhizobia du sol présentes dans les nodosités de leurs racines. Plusieurs études ont montré que la capacité de nodulation des légumineuses est fortement influencée par la fertilisation. L'objectif principal de cette étude est de promouvoir la pratique culturale permettant d'améliorer la capacité de nodulation des légumineuses

\section{MATERIEL ET METHODES Site d'étude}

La zone d'étude est située dans la région du Haut-Sassandra au Centre-Ouest de la Cote d'Ivoire dans le département de Daloa. Le département de Daloa situé entre 6 ${ }^{\circ} 53^{\prime} 58^{\prime}$ ' de latitude Nord et $6^{\circ} 26^{\prime} 32^{\prime \prime} \mathrm{W}$ de longitude Ouest. Cette zone est sujette à quatre saisons répartis comme suit: une grande saison des pluies allant d'avril à mi-juillet, une petite saison sèche de mi-juillet à mi-septembre, une petite saison des pluies de mi-septembre à novembre et la grande saison sèche de décembre à mars.

\section{Matériel végétal}

Le matériel végétal était constitué de semences de deux espèces de légumineuses herbacées alimentaires. Il s'agit des graines de haricot mungo (Vigna radiata) appelé communément soja vert de couleur verte et celles du niébé (Vigna unguiculata) achetées sur le marché de la ville de Daloa dans le mois de mars.

\section{Dispositif expérimental et collecte des données}

Le champ expérimental a été installé sous forme d'une pépinière couvrant un espace de $12 \mathrm{~m}^{2}$. Il était composé de deux blocs. Le premier représentant l'une des espèces et le second bloc représentant l'autre espèce. Au sein de chaque bloc, il y avait deux parcelles élémentaires séparé de $0,3 \mathrm{~m}$. Les deux blocs ont été espacés de 0,5 m. Pour chaque espèce, il a été appliqué 6 traitements avec 3 répétitions. Il s'agit des traitements T1 : sans apport de fertilisants ; T2 : apport d'azote sous forme d'urée; T3 : apport de phosphore sous forme de triple superphosphate ; T4 : apport d'un mélange azote-phosphate dans les mêmes proportions ; T5 : apport d'engrais complexe NPK ; T6 : sol amendé avec de l'engrais organique sous 
forme de compost. L'évaluation de la capacité de nodulation a été faite en considérant deux paramètres après la floraison. Il s'agit du nombre et du poids des nodules. Les mesures ont été effectuées sur toutes les plantes de la pépinière selon le type de fertilisant. Ces données ont été prises environ 55 jours après semis. Le nombre de nodules (NbNod) a été noté pour chaque plante. Le poids des nodules (PoNod) a été également déterminé.

\section{Analyse statistique}

Pour chaque variable étudiée (le nombre de nodules et le poids des nodules), les moyennes ont été comparées en prenant en compte les espèces et les types de fertilisation à travers une analyse de la variance à deux facteurs (ANOVA 2). La signification du test a été déterminée en comparant la probabilité (P) associée à la statistique au seuil $\alpha=0,05$. Lorsqu'une différence significative a été observée entre les caractères, l'ANOVA a été complétée par le test de la Plus Petite Différence Significative (PPDS). La PPDS permet de voir les groupes homogènes, étant donné qu'elle nous situe à quel niveau cette différence significative a lieu. Le logiciel statistique utilisé dans le cadre de ce travail est STATISTICA version 7.1. Les résultats sont présentés sous la forme moyennes plus ou moins écart-type.

\section{RESULTATS}

\section{Comparaison de la nodulation des deux espèces de légumineuses}

Les résultats de l'analyse statistique consignés dans le Tableau 1 n'ont montré aucune différence significative de l'effet espèce sur la capacité de nodulation des légumineuses $(\mathrm{P}>0,05)$ donnant des valeurs statistiquement identiques.

\section{Influence des fertilisants sur la capacité de nodulation des deux espèces}

Les fertilisants ont influencé de manière significative $(\mathrm{P}<0,05)$ les paramètres liés à la capacité de nodulation (Tableau 2). La fertilisation $6 \mathrm{~g}$ de compost a donné la valeur moyenne la plus élevée du nombre de nodules par rapport aux autres fertilisants. Cette valeur (147,25 nodules) est suivie de celle obtenue avec $9 \mathrm{~g}$ de compost qui est de 86,08 nodules. Comparativement au témoin (46 nodules), la dose de $6 \mathrm{~g}$ de compost a produit environ 3 fois plus de nodules, son poids nodulaire étant le poids le plus élevé $(2,70 \mathrm{~g})$. Par contre la valeur la plus faible a été observée avec les fertilisations azotées simples et combinées avec $3 \mathrm{~g}$ d'azote. On observe également une augmentation du nombre de nodules avec l'accroissement de la dose de phosphore (1g P, 2 g P, 3 g P). Cependant, l'augmentation des doses d'azote a entrainé une baisse du nombre et du poids des nodules. Une augmentation des valeurs moyennes des paramètres étudiés est également obtenue en faisant croître les doses $\mathrm{du}$ fertilisant organique jusqu'à $6 \mathrm{~g}$. Au-delà de cette dose, on assiste à une diminution de ces valeurs.

\section{Effet de l'interaction espèce-fertilisants sur la nodulation des deux espèces}

Le Tableau 3 montre l'effet de l'interaction espèce-dose de fertilisants sur la capacité de nodulation. Il y'a un effet significatif $(\mathrm{P}<0,05)$ de l'action combinée de ces deux facteurs sur les paramètres étudiés. La nodulation la plus forte est obtenue avec l'interaction haricot mungo-6 $\mathrm{g}$ de compost avec un nombre de nodules de 197,50 nodules par plant. Cependant, la plus petite valeur est issue du traitement haricot mungo- $2 \mathrm{~g} \mathrm{~N}$ et $3 \mathrm{~g}$ $\mathrm{N}$. Pour ces deux traitements, aucune espèce n'a nodulé. Le poids des nodules le plus élevé a été obtenu avec le les deux espèces à $6 \mathrm{~g}$ de compost. Ce poids est de l'ordre de 2,7 g par plant. Le plus faible poids obtenu avec les interactions qui n'ont présenté aucun nodule (haricot-mungo associé à $2 \mathrm{~g}$ de $\mathrm{N}$ et $3 \mathrm{~g}$ de $\mathrm{N})$. 
Tableau 1: Nombre et poids des nodules de deux espèces de légumineuses Vigna radiata L.Wilczek et Vigna unguiculata L.Walp (Fabaceae).

\begin{tabular}{llclc}
\hline Variables & & \multicolumn{2}{c}{ espèces } & \multicolumn{2}{c}{ Paramètres du test } \\
\hline \multirow{2}{*}{ NbNod/plt } & haricot mungo & F & P \\
\cline { 2 - 5 } PoNod/plt $(\mathbf{g})$ & $62,98 \pm 43,15^{\mathrm{a}}$ & $75,54 \pm 81,42^{\mathrm{a}}$ & $\mathbf{1 , 7 8}$ & $\mathbf{0 , 1 8}$ \\
\hline & $0,94 \pm 0,96^{\mathrm{a}}$ & $1,01 \pm 1,40^{\mathrm{a}}$ & $\mathbf{0 , 1 5}$ & $\mathbf{0 , 6 9}$ \\
\hline
\end{tabular}

NbNod/plt : nombre de nodules par plant, PoNod/plt : Poids des nodules par plant, NB : Les valeurs sur les lignes suivies de la même lettre ne sont pas significativement différentes selon le test de la Plus Petite Différence Significative (PPDS) à 5\% de probabilité.

Tableau 2: Nombre et poids des nodules de deux espèces de légumineuses Vigna radiata L. Wilczek et Vigna unguiculata L. Walp (Fabaceae) selon les doses et types de fertilisants.

\begin{tabular}{|c|c|c|}
\hline Doses de fertilisants & NbNod/plt & PoNod/plt (g) \\
\hline $1 \mathrm{~g} \mathrm{~N}$ (urée) & $22,25 \pm 17,77^{\mathrm{a}}$ & $0,23 \pm 0,24^{\mathrm{a}}$ \\
\hline 2 g N (urée) & $22,16 \pm 10,94^{\mathrm{a}}$ & $0,35^{ \pm} 0,38^{\mathrm{a}}$ \\
\hline 3 g N (urée) & $21,00 \pm 10,78^{\mathrm{a}}$ & $0,13 \pm 0,16^{\mathrm{a}}$ \\
\hline $1 \mathrm{~g} \mathrm{P}$ (Triple superphosphate) & $39,75 \pm 19,18^{\mathrm{b}}$ & $0,45 \pm 0,45^{\mathrm{b}}$ \\
\hline 2 g P (Triple superphosphate) & $40,83 \pm 26,94^{\mathrm{b}}$ & $0,29 \pm 0,22^{\mathrm{a}}$ \\
\hline 3 g P (Triple superphosphate) & $46,16 \pm 10,54^{\mathrm{c}}$ & $0,12 \pm 0,16^{\mathrm{a}}$ \\
\hline $1 \mathrm{~g} \mathrm{NP}$ & $44,66 \pm 43,65^{\mathrm{c}}$ & $0,24 \pm 0,20^{\mathrm{a}}$ \\
\hline $2 \mathrm{~g} \mathrm{NP}$ & $57,16 \pm 58,37^{\mathrm{d}}$ & $0,17 \pm 0,16^{\mathrm{a}}$ \\
\hline $3 \mathrm{~g} \mathrm{NP}$ & $22,66 \pm 11,58^{\mathrm{a}}$ & $0,10 \pm 0,07^{\mathrm{a}}$ \\
\hline $3 \mathrm{~g}$ compost & $83,50 \pm 38,42^{\mathrm{e}}$ & $1,36 \pm 0,56^{\mathrm{d}}$ \\
\hline $6 \mathrm{~g}$ compost & $147,25 \pm 65,61^{\mathrm{f}}$ & $2,70 \pm 1,01^{\mathrm{g}}$ \\
\hline $9 \mathrm{~g}$ compost & $86,08 \pm 65,29^{e}$ & $1,72 \pm 1,02^{\mathrm{e}}$ \\
\hline Témoin & $46,00 \pm 30,43^{c}$ & $0,79 \pm 0,37^{\mathrm{c}}$ \\
\hline \multirow{2}{*}{$\begin{array}{l}\text { Paramètres } \\
\text { du test }\end{array}$} & 13,5 & 22,82 \\
\hline & $<0,001$ & $<0,001$ \\
\hline
\end{tabular}

N : Azote, P : Phosphore, NP: Azote-Phosphore, NPK: engrais minéral complet, NbNod/plt: nombre de nodules par plant, PoNod/plt: Poids des nodules par plant, NB : Les valeurs dans les colonnes suivis de même lettre ne sont pas significativement différents selon le test de la Plus Petite Différence Significative (PPDS) à 5\% de probabilité. 
Tableau 3: Nombre et poids des nodules de deux espèces de légumineuses Vigna radiata L. Wilczek et Vigna unguiculata L. Walp (Fabaceae) selon l'interaction espèces-fertilisants.

\begin{tabular}{|c|c|c|c|}
\hline Espèces & Fertilisants & NbNod/plt & PoNod/plt (g) \\
\hline Haricot mungo & 1g N (urée) & $9,33 \pm 9,99^{b}$ & $0.16 \pm 0,29^{b}$ \\
\hline Haricot mungo & 2 g N (urée) & $0^{\mathrm{a}}$ & $0^{\mathrm{a}}$ \\
\hline Haricot mungo & $3 \mathrm{~g} \mathrm{~N}$ (urée) & $0^{\mathrm{a}}$ & $0^{\mathrm{a}}$ \\
\hline Haricot mungo & 1 g P (TSP) & $39,50 \pm 22,19 \mathrm{~S}^{\mathrm{b}}$ & $0,40 \pm 0,15^{\mathrm{b}}$ \\
\hline Haricot mungo & 2 g P (TSP) & $35,16 \pm 34,70^{\mathrm{b}}$ & $0,14 \pm 0,13^{\mathrm{b}}$ \\
\hline Haricot mungo & 3 g P(TSP) & $31,00 \pm 47,11^{\mathrm{b}}$ & $0,02 \pm 0,03^{\mathrm{a}}$ \\
\hline Haricot mungo & $1 \mathrm{~g} \mathrm{NP}$ & $39,16 \pm 63,03^{b}$ & $0,12 \pm 0,11^{\mathrm{b}}$ \\
\hline Haricot mungo & $2 \mathrm{~g} \mathrm{NP}$ & $71,00 \pm 81,08^{c}$ & $0,07 \pm 0,07^{\mathrm{b}}$ \\
\hline Haricot mungo & $3 \mathrm{~g} \mathrm{NP}$ & $5,33 \pm 7,14^{\mathrm{a}}$ & $0,05 \pm 0,07^{\mathrm{a}}$ \\
\hline Haricot mungo & $3 \mathrm{~g}$ compost & $108,67 \pm 30,63^{\mathrm{e}}$ & $1,37 \pm 0,42^{\mathrm{d}}$ \\
\hline Haricot mungo & $6 \mathrm{~g}$ compost & $197,50 \pm 49,85^{\mathrm{j}}$ & $2,61 \pm 1,33^{\mathrm{g}}$ \\
\hline Haricot mungo & $9 \mathrm{~g}$ compost & $111,33 \pm 85,79^{\mathrm{e}}$ & $1,52 \pm 0,98^{\mathrm{cd}}$ \\
\hline Haricot mungo & Témoin & $58,83 \pm 38,34^{\mathrm{ib}}$ & $0,96 \pm 0,32^{\mathrm{c}}$ \\
\hline Niébé & 1g N (urée) & $35,16 \pm 13,96^{\mathrm{b}}$ & $0,30 \pm 0,19^{\mathrm{b}}$ \\
\hline Niébé & 2 g N (urée) & $44,33 \pm 13,77^{\mathrm{b}}$ & $0,71 \pm 0,14^{\mathrm{c}}$ \\
\hline Niébé & 3 g N (urée) & $42,00 \pm 13,63^{b}$ & $0,26 \pm 0,14^{\mathrm{b}}$ \\
\hline Niébé & $1 \mathrm{~g} \mathrm{P}$ (TSP) & $40,00 \pm 17,81^{\mathrm{b}}$ & $0,50 \pm 0,34^{\mathrm{ab}}$ \\
\hline Niébé & 2 g P (TSP) & $46,50 \pm 17,75^{\mathrm{b}}$ & $0,45 \pm 0,17^{\mathrm{b}}$ \\
\hline Niébé & 3 g P (TSP) & $61,33 \pm 44,66^{\mathrm{ib}}$ & $0,23 \pm 0,17^{b}$ \\
\hline Niébé & $1 \mathrm{~g} \mathrm{NP}$ & $50,16 \pm 12,12^{b}$ & $0,35 \pm 0,20^{\mathrm{b}}$ \\
\hline Niébé & $2 \mathrm{~g} \mathrm{NP}$ & $43,33 \pm 24,96^{\mathrm{b}}$ & $0,28 \pm 0,13^{\mathrm{b}}$ \\
\hline Niébé & $3 \mathrm{Gnp}$ & $40,00 \pm 30,02^{b}$ & $0,13 \pm 0,03^{\mathrm{b}}$ \\
\hline Niébé & $3 \mathrm{~g}$ compost & $58,33 \pm 28,10^{\mathrm{ab}}$ & $1,35 \pm 0,71^{\mathrm{d}}$ \\
\hline Niébé & $6 \mathrm{~g}$ compost & $97,00 \pm 30,43^{\mathrm{cd}}$ & $2,78 \pm 0,68^{\mathrm{g}}$ \\
\hline Niébé & $9 \mathrm{~g}$ compost & $60,83 \pm 22,08^{\mathrm{ab}}$ & $1,93 \pm 1,10^{\mathrm{e}}$ \\
\hline Niébé & Témoin & $33,16 \pm 30,16^{\mathrm{b}}$ & $0,61 \pm 0,43^{\mathrm{ac}}$ \\
\hline \multirow[t]{2}{*}{ Paramètre du test } & $\mathbf{F}$ & 3,4 & 2,68 \\
\hline & $\mathbf{P}$ & $<0,001$ & $<0,001$ \\
\hline
\end{tabular}

N: Azote, P: Phosphore, TSP : Triple superphosphate, NP: Azote-Phosphore, NPK: engrais complet, NbNod/plt: nombre de nodules par plant, PoNod/plt: Poids des nodules par plant, NB : Les valeurs dans les colonnes suivies de même lettre ne sont pas significativement différentes selon le test de la Plus Petite Différence Significative (PPDS) à 5\% de probabilité. 


\section{DISCUSSION}

Les résultats des travaux effectués montrent que le nombre et le poids des nodules ne dépendent pas des deux espèces de légumineuses cultivées. En effet, la capacité de nodulation des légumineuses reste constante d'une espèce à une autre. Ces résultats sont contradictoires à ceux de Muhammad et al. (2010) qui ont montré que le nombre de nodules observé au niveau du soja était significativement différent de celui du niébé. Les variations pourraient probablement s'expliquer par la fertilité native du sol (Sanginga et al., 1997), les souches indigènes de rhizobia et les techniques culturales (Okogun et al., 2005). Cela pourrait également s'expliquer par la différence au niveau de la phénologie et les besoins des deux espèces. Une étude similaire menée sur quatre espèces de légunineuses (Medicago ruthenica, Medicago sativa, Medicago falcata, Lespedeza daurica) par $\mathrm{Li}$ et al. (2015) sous serre a montré que le nombre de nodules et leurs poids variaient d'une espèce à une autre avec des différences hautement significatives. Cette différence serait liée aux conditions abiotiques du milieu de culture telles que la température et l'eau.

Le nombre de nodules et le poids des nodules dépendent du type de fertilisant épandu. Konthoujam et al. (2013) ont obtenu les mêmes résultats sur le nombre et le poids des nodules. L'augmentation relative du poids des nodules pourrait être attribué au nombre de nodules produits par plant. La fertilisation organique a permis d'accroître aussi la capacité de nodulation à travers l'augmentation du nombre et du poids des nodules. Cette augmentation du nombre et du poids des nodules pourrait s'expliquer par le fait que l'ajout de fertilisants organiques au sol accroît la matière organique, l'azote et le phosphore total affectant ainsi la communauté microbienne du sol (Dong et al. 2014). La population microbienne augmente ainsi considérablement entrainant la multiplication du nombre de nodules. En somme, les intrants organiques aident à améliorer la capacité du sol à maintenir l'eau, un facteur essentiel dans le processus de nodulation. Les intrants organiques contribuent à la croissance des racines et le contrôle des propriétés physiques et chimiques des sols qui influent sur l'absorption et l'accumulation des éléments nutritifs du sol. Aussi les intrants organiques aident à ajouter des nutriments qui sont absents dans les engrais minéraux. Ils créent ainsi un environnement favorable pour le développement des racines, améliorent l'accessibilité des racines des plantes au phosphore en rendant le nutriment disponible, et réduisent le stress des cultures liées aux facteurs comme l'acidité du sol (Fairhurst, 2012). Ce résultat est en conformité avec celui de Yeboah (2013) dont les travaux ont prouvé une augmentation du nombre et du poids de nodules obtenus avec l'apport de fertilisant organique sous forme de fiente de poulet.

Nos résultats dénotent une faible nodulation en présence de la fertilisation azotée (urée). Selon Uddin et al. (2008), le nombre et la taille des nodules sont considérablement contraints par l'utilisation d'engrais azotée. Voisin et al. (2010) affirment que lorsque la nodulation a lieu, le nombre de nodosités mis en place au sein du système racinaire de la légumineuse est proportionnel aux besoins en azote de la plante pour sa croissance. La fixation symbiotique démarre dès que les réserves de la graine et les reliquats azotés ne permettent plus de subvenir aux besoins en azote pour la croissance (Voisin et al., 2010). Cependant, Razzaque et al. (2016) ont prouvé par leurs travaux une augmentation du nombre de nodules en présence d'engrais azoté. Ils expliqueraient cela par une action stimulatrice sur le développement et la croissance de la plante. Cela pourrait avoir un effet positif sur la nodulation (Akbari et al., 2008). Nos travaux ont montré que le nombre de nodules augmentent avec l'accroissement de la dose de phosphore. En effet, selon Qiao et al. (2007), l'apport de phosphore augmente la quantité d'azote fixée par les nodules et sa concentration dans la plante. 


\section{Conclusion}

Il ressort de cette étude qu'il existe une abondance souches de rhizobiums capables d'exprimer une grande affinité à ces deux espèces de légumineuses étudiées. Les fertilisants minéraux et organiques affectent la capacité des deux légumineuses à fixer une grande quantité d'azote atmosphérique. Cependant, l'épandage au semis des engrais azotés comme l'urée a inhibé la nodulation. La fertilisation organique à la dose de $6 \mathrm{~g}$ par plant pourrait être conseillée pour un meilleur accroissement de la capacité de nodulation

\section{CONFLIT D'INTERETS}

Les auteurs ne déclarent aucun conflit d'intérêts.

\section{CONTRIBUTIONS DES AUTEURS}

Pour ces travaux, JKN, NK et JKY ont réalisé l'échantillonnage tout entier et pris part dans le traitement des données et l'élaboration du document final. KA a supervisé les travaux. JKY a mis à la disposition de l'équipe son laboratoire de recherche et fourni le matériel de travail.

\section{REMERCIEMENTS}

Nos remerciements vont à l'endroit des Enseignants et des étudiants du Laboratoire d'amélioration de la production agricole de l'Université Jean Lorougnon GUEDE de Daloa, UFR Agroforesterie pour les efforts consentis dans la réalisation de ces travaux.

\section{REFERENCES}

Akbari N, Barani M, Ahmadi H. 2008. Change of grain protein content and correlations with other characteristics under planting pattern and starter $\mathrm{N}$ fertilizer of mungbean (Vigna radiata $\mathrm{L}$. Wilczek). Am. Eurasian Journal Agriculture Environment Sciences, 4: 306-310.

Dong WI, Zhang XY, Dai XQ, Fu XL, Yang FT, Liu XY, Sun XM, Wena XF, Schaeffer S. 2014. Changes in soil microbial community composition in response to fertilization of paddy soils in subtropical China Applied Soil Ecology, 84: $\quad 140-147 . \quad$ DOI: https://doi.org/10.1016/j.apsoil.2014.06. 007

Fairhurst T. 2012. Handbook for Integrated Soil Fertility Management. Africa Soil Health Consortium, Nairobi p. 7.

Floret C, Pontanier R. 2000. La jachère en Afrique tropicale. Rôle, Aménagement, Alternatives. Vol I, Actes de séminaire international, Dakar, 13-16 avril 1999. U.E., CORAF, IRD. Edition John Libbey Eurotext, Paris, p. 777.

Graveline N, Loubier S. 2004. Instruments de contrôle de la pollution agricole par les nitrates : revue de littérature. Bureau de recherches géologiques et minières (BRGM).

Kasongo RK, Van Ranst E, Kanyankogote P, Verdoodt A, Baert G. 2012. Réponse du soja (Glycine max) à l'application de phosphate de Kanzi et de dolomie rose de Kimpese sur sol sableux en RD Congo. Canadian Journal of Soil Science, 92: 905-916. DOI: 10.4141/CJSS2011-097

Konthoujam ND, Singh TB, Athokpam HS, Singh NB, Shamurailatpam D. 2013. Influence of inorganic, biological and organic manures on nodulation and yield of soybean (Glycine max Merril L.) and soil properties. Australian Journal of Crop Sciences, 7(9): 1407-1415. DOI: 10.21475/ajcs.2013

Li Q, Song Y, Li G, Wang P, Zhou D. 2015. Grass-legume impact soil N, species recruitment, and productivity in temperate steppe grassland. Plant and Soil, 394: 271-285. DOI: https://doi.org/10.1007/s11104-0152525-2

Muhammad A, Dikko AU, Audu M, Singh A. 2010. Comparative Effects of Cowpea and Soybean Genotypes on N2 - Fixation and N-Balance in Sokoto Dry SubHumid Agro-Ecological Zone of Nigeria. Nigerian Journal of Basic and Applied 
Science, 18(2): 297-303. DOI: http://dx.doi.org/10.4314/njbas.v18i2.64 346

Okogun OO, Sanginga N, Abaidoo R, Dashiell KE, Diels J. 2005. On-farm evaluation of biological nitrogen fixation potential and grain yield of Lablab and two soybean varieties in the northern Guinea savanna of Nigeria. Nutrient Cycling in Agroecosystems, 73: 265-275. DOI: $10.1007 / \mathrm{s} 10705-005-3821-7$

Qiao WH, ZhaoXY, LI W, Zhang XY. 2007. Overexpression of AeNHX1, a rootspecific vascuolar $\mathrm{Na}+/ \mathrm{H}+$ Antiporter from agropyron elongatum, confers salt tolerance to Arabidopsis and festuca plants. Plant Cell Reports (26-9): 166372. DOI: $10.1007 / \mathrm{s} 00299-007-0354-3$

Razzaque MA, Haque MM, Karim MA, Solaiman ARM. 2016. Nitrogen fixating ability of mungbean genotypes under different levels of nitrogen application. Bangladesh J. Agril. Res., 41(1): 163171. DOI: $10.3329 /$ bjar.v41i1.27681

Sanginga N, Dashiell K, Okogun JA, Thottappilly G. 1997. Nitrogen and N Contribution by promiscuous nodulating soybeans in the southern guinea savanna of Nigeria. Plant and Soil, 195: 257-266. DOI:https://doi.org/10.1023/A:10042075 30131.
Uddin MB, Khan MAS, Mukul SA, Hossain MK. 2008. Effects of inorganic fertilizers on biological nitrogen fixation and seedling growth of some agroforestry trees in Bangladesh. Journal of Forestry Research, 19(4): 303-306. DOI: $10.1007 / \mathrm{s} 11676-008-0054-\mathrm{Z}$

Voisin AS, Munier-Jolain NG, Salon C. 2010. The nodulation process is tightly linked to plant growth. An analysis using environmentally and genetically induced variation of nodule number and biomass in pea. Plant and Soil, 337: 399412.

DOI:

https://doi.org/10.1007/s11104-0100536-6

Yeboah G. 2013. Effects of NPK and poultry manure rates on the growth, nitrogen fixation and grain yield of soybean (Glycine max (L) Merrill). Thesis for Master degree BSc.Natural Resources Management (Hons); DipEd., Faculty of Agriculture of the College of Agriculture and Natural Resources, Kwame Nkrumah University of Science and Technology, Kumasi, Ghana, p. 57. 